\title{
A CASE STUDY ON HOW DISTANT TREES APPEAR TO BE MOVING IN THE SAME DIRECTION AS OUR RIDE
}

\author{
Omkar Devidas Kadam \\ Fourth year, Undergraduate Student, Mechanical Engineering \\ IIT Gandhinagar, Village Palaj, Gandhinagar, Gujarat -382355, India
}

\begin{abstract}
It is of common observation that, when we ride on a bus, train, or vehicle, the trees alongside seem to travel in the direction opposite to ours. This is on account of the relative motion between us and the trees. Interestingly, it is the motion of the trees that are far away from the road that appears strange. The trees that are far away when observed in the presence of other objects in the foreground almost give the illusion that they are moving along with us in the same direction of the ride. Realizing that not many people are aware of this illusion and hardly there is any illustration to explain it, in what follows, I discussed how this perception could be explained with the help of basic geometry and motion analysis. We refer to this illusion as 'false relative motion'.
\end{abstract}

Keywords - relative motion, perception, false relative motion

\section{INTRODUCTION}

We consider the situation in which the observer (point $O$ in Fig 1) is traveling along a straight road $O A^{\prime}$. A distant tree $B$ is being observed by the observer, while the other trees or features closer are still in view (tree A, for example). In this note, we seek to show that $B$ will be perceived to move in the same direction as the observer when there are other features or trees present closer to the road, such as $A$. We first set up this problem with appropriate conventions as below.

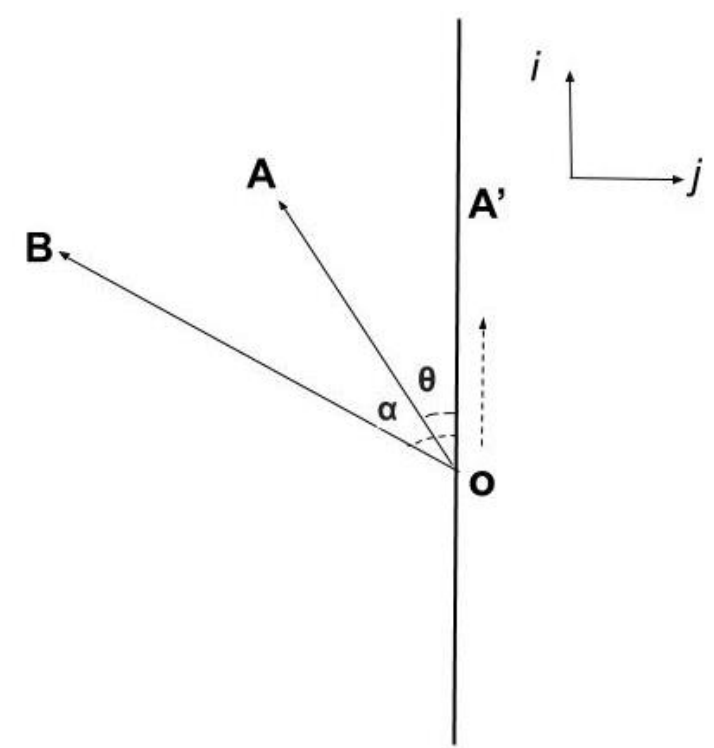

Fig. 1. Schematic to explain observed false relative motion.

As shown in Fig $1, i, j$ and $k$ be the right handed orthogonal vectors. At time $t$, let $A$ and $B$ represent two trees in the open field alongside the road and $O$ represents the observer's position on the road. Let the observer be traveling towards $A^{\prime}$ from $O$ with a constant velocity $v$. Please note that, $i \| O A^{\prime}, \angle B O A^{\prime}=\alpha, \angle A O A^{\prime}=\theta, l(B O)=R_{2}$ and $l(A O)=R_{1}$.

\section{A. Analysis -}

Below we figure out condition under which false relative motion will be observed. 

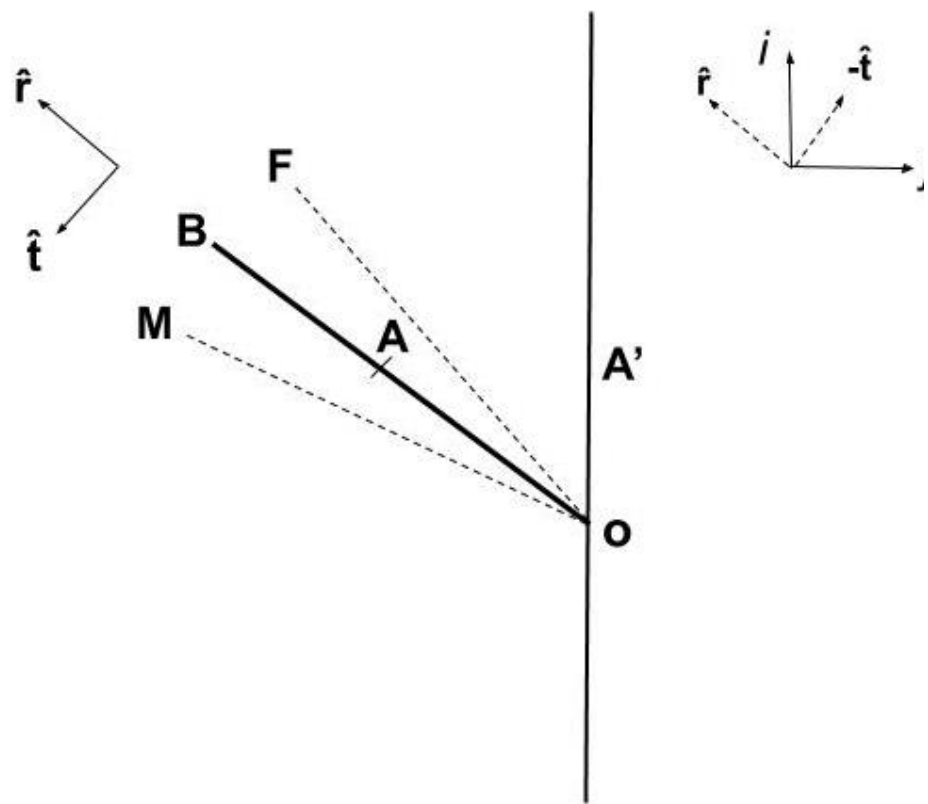

Fig. 2. $\angle$ FOM represents the field of view of the observer $O$.

In Fig 2, we define a vector $\hat{r}$ in the direction $\widehat{A B}$ and a vector $\hat{t}$ perpendicular to it. The region represented by $\angle \mathrm{FOM}$ is a region the observer is able to observe. Let $B$ represents a tree very far from the observer $O$. While observing tree $B$, the observer is focusing on a small region (region is represented by a dark line BAO in Fig 2). Next, let us look at the situation in which the tree $A$ enters into this small region of focus causing $\alpha \approx \theta$. In this case, we derive the instantaneous velocity of the distant tree $B$ observed by the observer $O$.

Considering $O$ as the origin, we can represent trees $A$ and $B$ as $\overrightarrow{R_{1}}$ and $\overrightarrow{R_{2}}$ respectively.

$$
\overrightarrow{A B}=l(A B) \hat{r}
$$

From Fig 1

$$
l(A B)=\sqrt{R_{1}{ }^{2}+R_{2}{ }^{2}-2 R_{2} R_{1} \cos (\alpha-\theta)} .
$$

Differentiating (1) with respect to time,

$$
\frac{d \overrightarrow{A B}}{d t}=l(A B) \frac{d \hat{r}}{d t} .
$$

But, $\hat{r}$ and $\hat{t}$ would be observed as rotating vectors by the observer because the observer is in motion. From ${ }^{1}$ we have,

$$
\frac{d \hat{r}}{d t}=\vec{\omega} \times \hat{r} \text {. }
$$

While riding on a train, bus, or vehicle, when we focus our attention on distant objects, since there are other objects in the foreground, we perceive the motion of distant objects relative to the foreground nearer objects. In this context, assuming $R_{1}<R_{2}$, as the tree $B$ is farther away from the observer than the tree $A$ when the observer watches the tree $B$ moving, the observer actually perceives its motion with respect to the tree $A$. Hence for the observer, the perceived rate of change of $\angle \alpha$ seems to be $\dot{\alpha}-\dot{\theta}$.

As explained above the observer is watching the tree $B$ with respect to the tree $A$ hence,
So, (4) may now be written as

$$
\vec{\omega}=(\dot{\alpha}-\dot{\theta})(-\hat{k}) .
$$

$$
\frac{d \hat{r}}{d t}=(\dot{\alpha}-\dot{\theta}) \hat{t}
$$

From Fig 1, it is clear that

$$
\dot{\theta}=\frac{v \sin (\theta)}{R_{1}} \text { and } \dot{\alpha}=\frac{v \sin (\alpha)}{R_{2}} .
$$

Using (4), (5), (6) and (7), we may write (3) as

$$
\frac{d \overrightarrow{A B}}{d t}=l(A B) v\left(\frac{\sin (\theta)}{R_{1}}-\frac{\sin (\alpha)}{R_{2}}\right)(-\hat{\mathrm{t}}) .
$$

Next, let $\vec{v}_{(B / A) O}$ be the velocity of the tree $B$ with respect to the tree $A$ observed by an observer $O$.

Note that, $\frac{d \overrightarrow{A B}}{d t}=\vec{v}_{(B / A) O}$. Both the trees $A$ and $B$ are on the same side of the road and the tree $B$ is far from the observer hence, we have $\alpha \approx \theta$. Moreover, using (2) and substituting $d=\frac{R_{2}}{R_{1}}$, (8) may be written as

$$
\vec{v}_{(B / A) O}=\sqrt{d^{2}+1-2 d \cos (\alpha-\theta)} v \sin (\alpha)\left(1-\frac{1}{d}\right)(-\hat{\mathrm{t}}) .
$$

From (9), for $d>1, \vec{v}_{(B / A) O}$ is along $(-\hat{\mathrm{t}})$. Condition for a very long distance between the observer and the tree $B$ is shown in Fig 2. Hence, there is a component of $\vec{v}_{(B / A) O}$ along $i$ direction. But the observer knows that $i$ is the direction of the ride. Hence, this is how the observer feels that the trees that are far away moves in the direction of ride and the instantaneous velocity perceived by an observer can be obtained from (9). Moreover, if the situation is not as special as discussed above, from (8), it can be seen that false relative motion will be observed whenever below condition is satisfied,

$$
\frac{R_{2} \sin \theta}{R_{1} \sin \alpha}>1
$$

\section{CONCLUSION}

In this note, a phenomenon of false relative motion in which distant stationary objects are perceived to be moving in the same direction of the observer's ride was explained using simple geometry and kinematic analysis. This notion of false relative motion is also applicable in the case of perceived curvilinear motion.

\section{ACKNOWLEDGEMENT}

I would like to express my sincere gratitude to Prof. Harish P. M. and Sujay Kadam for their guidance and comments which greatly improved the manuscript.

\section{REFERENCES}

[1] Meriam J. L. and Kraig L. G., "Engineering Mechanics Dynamics, Seventh Edition", Wiley India, pp. 385-390, 2016. 